\title{
ARTICLE
}

\section{Radiation Response of a Semi-insulating GaAs Semiconductor Detector for Charged Particle at Variable Operating Temperature}

\author{
Sang Mook KANG ${ }^{1,2}$, Jang Ho HA ${ }^{1 *}$, Se-Hwan PARK ${ }^{1}$, Han Soo KIM ${ }^{1}$, Nam Ho LEE ${ }^{1}$, and Yong Kyun KIM ${ }^{2}$ \\ ${ }^{1}$ Korea Atomic Energy Research Institute, Daejon, 305-600, Korea \\ ${ }^{2}$ Department of Nuclear Engineering, Hanyang University, Seoul, 133-791, Korea
}

\begin{abstract}
GaAs is a wide band gap semiconductor $(1.42 \mathrm{eV})$ with potential use as a room temperature radiation detector. We have studied the radiation response of a prototype semiconductor detector by using a undoped semi-insulating (SI) GaAs wafer. The properties of the GaAs wafer are a resistivity of $10^{7} \mathrm{ohm}-\mathrm{cm}$, an orientation of (100) and a diameter of $50.8 \mathrm{~mm}$. The dimensions of the GaAs SI detectors were about $5 \times 5 \mathrm{~mm}^{2}$ with a 350 um thickness. Prior to a metallization process, the surfaces of the GaAs wafer were cleaned by a solvent and rinsed with de-ionized (DI) water, and the removal of an oxidation layer by a diluted acid solution was performed. Metal/semiconductor contacts on the surface were fabricated by using a thermal evaporator in a high vacuum condition. The prototype GaAs detector had circular metal contacts of $\mathrm{Ni} / \mathrm{Au}$ at each side and the diameter of a circular contact was $3 \mathrm{~mm}$. The current-voltage characteristics of GaAs semiconductor detector were measured by using HP parameter analyzer with voltage sources. We measured the leakage currents at different temperature conditions. Also, the radiation response was evaluated by Am-241 source at variable operating temperature.
\end{abstract}

\section{KEYWORDS: gallium arsenide, GaAs, semiconductor detector, radiation detector, charged particle}

\section{I . Introduction}

The last decade has seen a growing interest in semiconductor radiation detectors operated at room or nearly room temperature. Great efforts have been invested in the development of radiation detectors based on semi-insulating (SI) GaAs. The main reasons are as follows: (i ) high resistance against radiation damage; (ii) it possesses a good energy resolution, which relates to its active volume; (iii) such a detector also exhibits fast signal rise times, which results from a high mobility and drift velocity of charge carriers; (iv) its large band gap energy allows a SI GaAs detector to operate at room temperature. Other important features are a good technology base and low production and operating costs. ${ }^{(1-3)}$

An alpha particle monitoring method for the detection of ${ }^{238} \mathrm{Pu}$ and ${ }^{235} \mathrm{U}$ is becoming important in homeland security. Alpha measurement in a vacuum is known to provide a good resolution sufficient to separate an isotope abundance in nuclear materials. However, in order to apply it to a high radiation field like a spent fuel treatment facility, a nuclear material loading and unloading process in a vacuum is one of the great disadvantages ${ }^{(4)}$ Therefore, the main technical issue is to develop a detector for alpha detection at air condition and low power operation for integration type device. In this study we fabricated GaAs Schottky detector by using semi-insulating (SI) wafer and measured current-voltage characteristic curve and alpha response with $5.5 \mathrm{MeV}^{241} \mathrm{Am}$ source.

\section{Experiments}

\section{Fabrication of $\mathrm{GaAs}$ radiation detector}

Detector structures have been fabricated by SI GaAs wafers grown by the LEC (liquid encapsulated Czochralski) growth method. The undoped semi-insulating wafers were an orientation of (100) and a diameter of $50.8 \mathrm{~mm}$. Front surface was polished and the back surface was etched after a lapping. Resistivity was measured as a $7.58 \times 10^{7} \mathrm{Ohm}-\mathrm{cm}$ and the Hall electron mobility was a $6,800 \mathrm{~cm}^{2} / \mathrm{V}$-s. The dimensions of the GaAs SI bulk detectors were about $5 \times 5$ $\mathrm{mm}^{2}$ with a $350 \mu \mathrm{m}$ thickness. Prior to a metallization process, the surfaces of the GaAs wafer were cleaned by a solvent and rinsed with de-ionized (DI) water, and the removal of an oxidation layer by a diluted acid solution was performed. Metal contacts on the surface were fabricated by using a thermal evaporator in a vacuum condition. On the front and the back surface a layered structure of $\mathrm{Au} / \mathrm{Ni}$ was deposited in order to obtain Schottky contact. The thickness of the metallic layers is $200 \mathrm{~nm}, 30 \mathrm{~nm}$ for $\mathrm{Au}, \mathrm{Ni}$, respectively and the diameter of a circular contact was 3 $\mathrm{mm}$.

\section{I-V characteristic of GaAs radiation detectors}

To measure the current-voltage curve, the PCB layer was made of a teflon substrate with a $1 \mathrm{~mm}$ diameter electrical contact pad. The GaAs sample was fixed by a conducting epoxy onto the teflon substrate and contacted by a wire for the electrical characteristics and detection properties measurements. Fig. 1 shows the diagram and photography of fabricated GaAs detector. The I-V characteristics of the SI GaAs Schottky radiation detector were measured by using HP parameter analyzer in Oven. We took the measurement under a biased voltage from the -40 to $40 \mathrm{~V}$ range in $0.1 \mathrm{~V}$ intervals and at variable operating temperatures. 


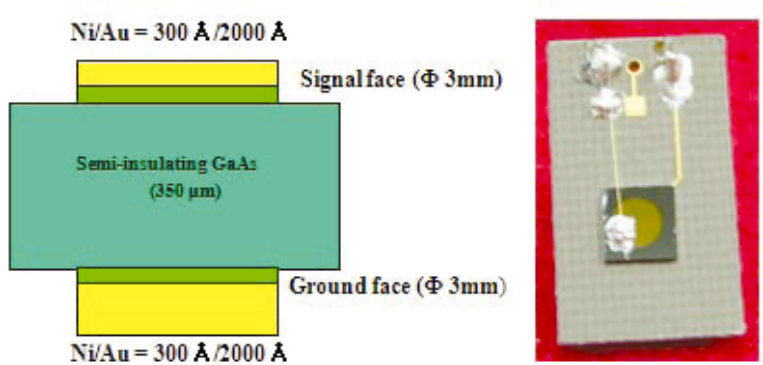

Fig. 1 The cross section diagram (left) and photography (right) of GaAs detector
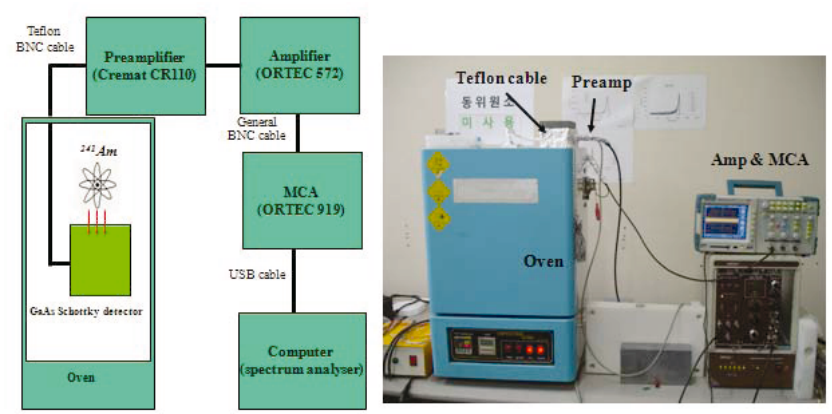

Fig. 2 The diagram (left) and photography (right) of experimental measurement system for alpha radiation response

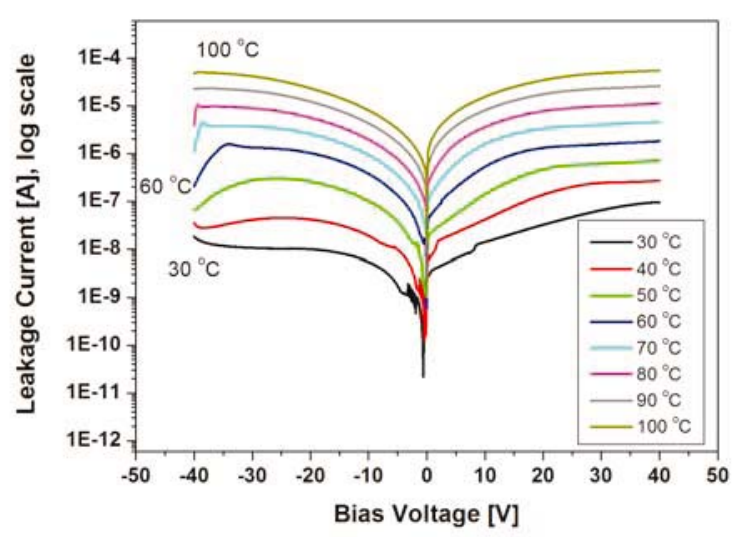

Fig. 3 The current-voltage characteristics curve as a function of bias voltage at various operating temperature.

\section{Alpha Response in room temperature}

The GaAs radiation detector was installed in a aluminum (Al) case to protect it from noise and the $\mathrm{Al}$ case was placed in Oven. Alpha response was evaluated by ${ }^{241} \mathrm{Am}$ source with $5.5 \mathrm{MeV}$ at room temperature in air. Alpha-particle spectra were obtained by a preamplifier based on a hybrid chip (CR110), ORTEC's shaping amplifier, a multi-channel analyzer (MCA) and DAQ.

\section{Alpha Response in variable operating temperature}

Fig. 2 shows measurement system for the alpha-particle spectra to obtain the temperature effect. The GaAs detector was installed in $\mathrm{Al}$ case for alpha response measurements in a high temperature atmosphere. The temperature range of the oven was $0 \sim 300^{\circ} \mathrm{C}$. A teflon $\mathrm{BNC}$ cable was used to connect the $\mathrm{Al}$ case with the preamplifier to persist in high temperature and pulse height spectra were obtained by using NIM standard electronics.

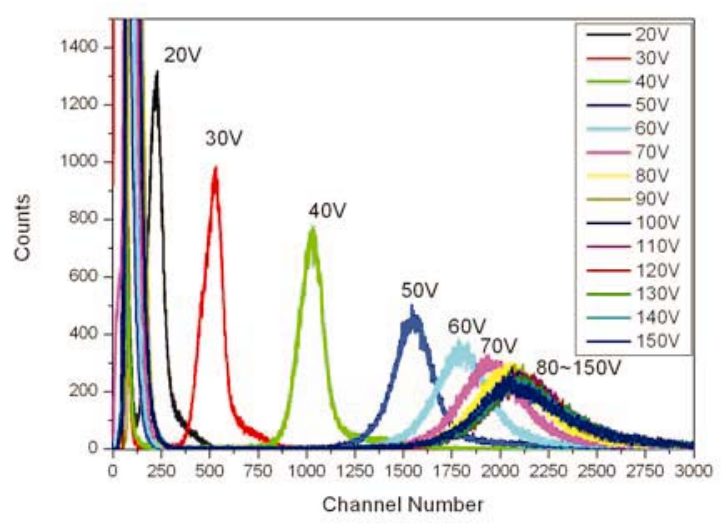

Fig. 4 The spectra with variable bias voltage at room temperature in air.

\section{Analysis}

The current-voltage characteristics were measured in the range from -40 to $40 \mathrm{~V}$ in $0.1 \mathrm{~V}$ intervals and at different temperatures. Fig. 3 shows the leakage current as a function of temperature. The leakage current was increased by about one order of magnitude for a $30{ }^{\circ} \mathrm{C}$ increase in temperature.

The alpha particles were normally incident through the anode, and a signal was extracted to the preamplifier via the cathode. Figure 4 shows the pulse height spectrums of the SI-GaAs Schottky detector by using $5.5 \mathrm{MeV}$ alpha particles from the ${ }^{241} \mathrm{Am}$ source. The spectrums were acquired with 20 to $150 \mathrm{~V}$ biased voltages in $10 \mathrm{~V}$ intervals, a $6 \mu \mathrm{s}$ shaping time and a $600 \mathrm{sec}$ collection time in an air condition and at room temperature. The source was placed approximately 3 $\mathrm{mm}$ from the surface of the GaAs detector. Clearly, there are full-energy peaks in the biased voltages.

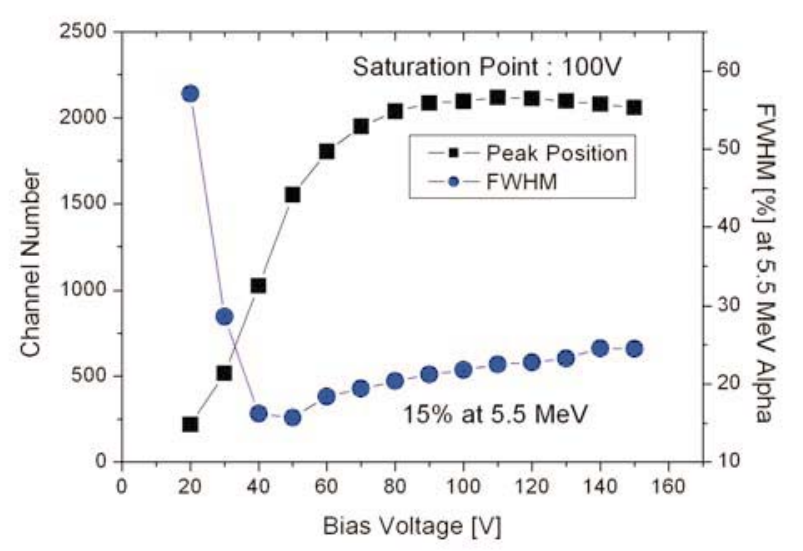

Fig. 5 The peak position and FWHM as a function of bias voltage at room temperature in air.

To determine the performance of the detector, we calculated its energy resolution in Full Width Half Maximum(FWHM) for $5.5 \mathrm{MeV}$ alpha particles from ${ }^{241} \mathrm{Am}$ source. An alpha energy resolution of $15 \%$ at $5.5 \mathrm{MeV}$ has been obtained at 
the biased voltage $50 \mathrm{~V}$. The saturation point of the peak position is bias voltage $100 \mathrm{~V}$. Generally, the standard depletion of the GaAs detector is about $1 \mu \mathrm{m} / \mathrm{V}$. Therefore, the fully depletion region of detector is about $100 \mu \mathrm{m}$.

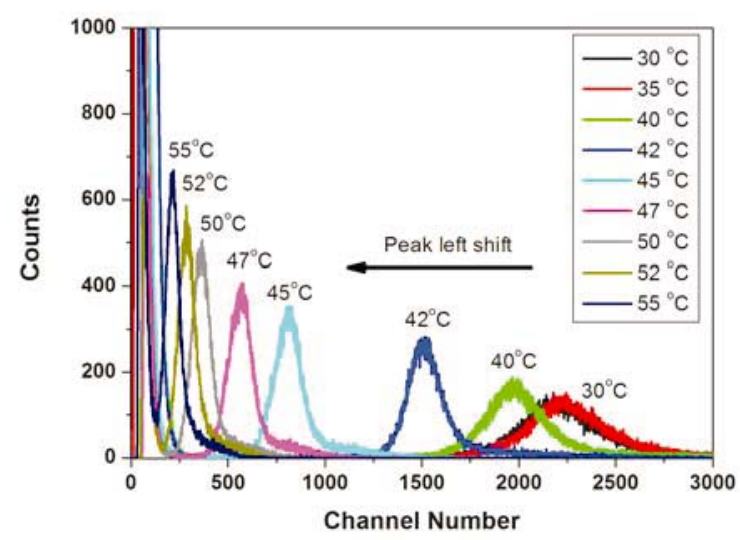

Fig. 6 The spectra of GaAs detector at biased voltage $100 \mathrm{~V}$ in various operating temperature.

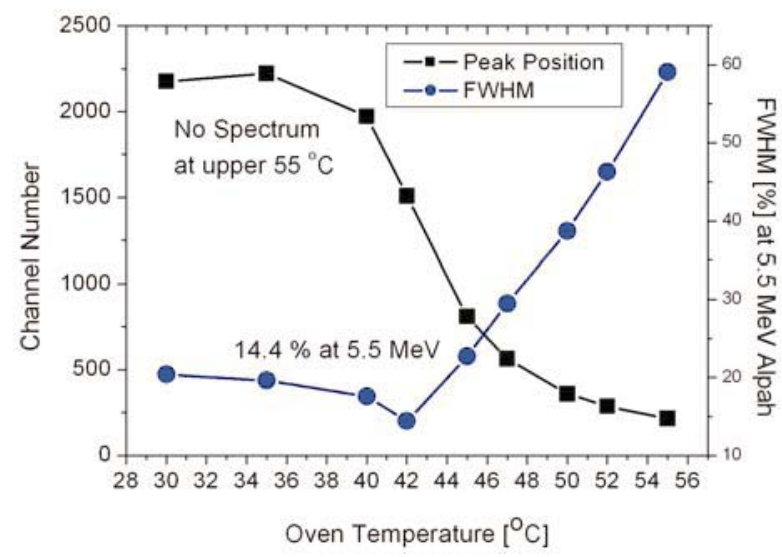

Fig. 7 The peak position and FWHM as a function of temperature in air.

Fig. 6 shows the spectra of detector in various operating temperature. The spectrums were acquired with $100 \mathrm{~V}$ biased voltage, a $6 \mu \mathrm{s}$ shaping time and a $600 \mathrm{sec}$ collection time in an air and at $30 \sim 55^{\circ} \mathrm{C}$ temperature. The peak position show the left shift with respect to temperature increase and the spectrum did not measured at upper $55^{\circ} \mathrm{C}$ temperature. An alpha energy resolution of $14.4 \%$ at 5.5 $\mathrm{MeV}$ has been obtained at the $42^{\circ} \mathrm{C}$ temperature (Fig. 7).

\section{Conclusion}

We fabricated GaAs Schottky detector by using semi-insulating (SI) wafer and measured current-voltage characteristics curve and alpha response with $5.5 \mathrm{MeV}$ ${ }^{241} \mathrm{Am}$ source. The leakage current was increased by about one order of magnitude for a $30^{\circ} \mathrm{C}$ increase in temperature. An alpha energy resolution of $15 \%$ at $5.5 \mathrm{MeV}$ has been obtained at the biased voltage $50 \mathrm{~V}$ at room temperature in air. The peak position show the left shift with respect to temperature increase and the spectrum did not measured at upper $55^{\circ} \mathrm{C}$ temperature. Also the energy resolution of $14.4 \%$ at $5.5 \mathrm{MeV}$ has been obtained at the biased voltage $100 \mathrm{~V}$ at $42^{\circ} \mathrm{C}$ in air.

\section{Acknowledgement}

This work has been carried out under the Nuclear R\&D program of the Ministry of Science and Technology (MOST) of Korea.

\section{References}

1) B. Zat'ko et al., "On the spectrometric performance limit of radiation detectors based on semi-insulating GaAs", Nucl. Instr. Meth. A, 531 (2004) 111

2) D. S. McGregor et al., "Room temperature compound semiconductor radiation detectors", Nucl. Instr. Meth. A, 395 (1997) 101

3) B. Zat'ko et al., "Performance of a Schottky suface barrier radiation detector based on bulk undoped semi-insulating GaAs at reduced temperature", IEEE Trans. Nucl. Scie. 53(2006) 625

4) Jang Ho Ha et al., "Fabrication and Characteristic of a fully depleted semi-insulating GaAs semiconductor detector for alpha radiation detection at room temperature in Air", J. Kor. Phy. Soci. 52(2008) 576 